\title{
BMJ open Use of non-steroidal anti-inflammatory drugs and proton pump inhibitors in correlation with incidence, recurrence and death of peptic ulcer bleeding: an ecological study
}

\author{
Yunxia Lu, ${ }^{1}$ Emma Sverdén, ${ }^{2}$ Rickard Ljung, ${ }^{1,3}$ Claes Söderlund, ${ }^{2}$ \\ Jesper Lagergren ${ }^{1,4}$
}

To cite: Lu Y, Sverdén E, Ljung $R$, et al. Use of nonsteroidal anti-inflammatory drugs and proton pump inhibitors in correlation with incidence, recurrence and death of peptic ulcer bleeding: an ecological study. BMJ Open 2013;3:e002056. doi:10.1136/bmjopen-2012002056

- Prepublication history for this paper are available online. To view these files please visit the journal online (http://dx.doi.org/10.1136/ bmjopen-2012-002056).

Received 4 September 2012 Revised 31 October 2012 Accepted 5 November 2012

This final article is available for use under the terms of the Creative Commons Attribution Non-Commercial 2.0 Licence; see http://bmjopen.bmj.com

For numbered affiliations see end of article

\section{Correspondence to}

Dr Yunxia Lu;

yunxia.lu@ki.se

\section{ABSTRACT}

Background: Non-steroidal anti-inflammatory drugs (NSAIDs) and proton pump inhibitors (PPIs) are regarded as two types of drugs that respectively increase and decrease the risk of peptic ulcer bleeding. However, their relation to occurrence, recurrence and death of bleeding in the population level is not clear.

Study objective: To clarify recent calendar-time correlations between sales of NSAIDs and PPIs and the occurrence of peptic ulcer bleeding, re-bleeding and death.

Design: Ecological study.

Results: The time trend of peptic ulcer bleeding did not correlate with PPI sales but did correlate with NSAIDs in mem $\left(R_{\text {male }}=0.6571, P_{\text {male }}=0.05\right)$. Sales of PPIs (inverse) and NSAIDs correlated with re-bleeding in women $\left(R_{\text {male }}=-0.8754, P_{\text {male }}=0.002\right.$ and

$R_{\text {female }}=0.7161, P_{\text {female }}=0.03$, respectively), but not in men. An inverse correlation between PPI sales and 30day death after bleeding was found $\left(R_{\text {male }}=-0.9392\right.$, $P_{\text {male }}=0.0002$ and $R_{\text {female }}=-0.8561, P_{\text {female }}=0.003$ ), and NSAID sales were found to correlate with increased death after bleeding $\left(\left(\mathrm{R}_{\text {male }}=0.7278, \mathrm{P}_{\text {male }}=0.03\right.\right.$,

$\left.\mathrm{R}_{\text {female }}=0.7858, \mathrm{P}_{\text {female }}=0.01\right)$.

Conclusions: The sales of NSAIDs and PPIs correlate with recurrence of peptic ulcer bleeding in women and death after peptic ulcer bleeding in both genders in the population level.

\section{INTRODUCTION}

Bleeding is one of the most frequent and serious complications of peptic ulcer disease. Despite the marked decrease in the occurrence of peptic ulcer disease during recent decades, paralleling the decreasing prevalence of Helicobacter pylori infection, the incidence of peptic ulcer bleeding has not changed apparently. On the contrary, several surveys have shown that the incidence of peptic ulcer bleeding has increased among older people. ${ }^{1-4}$

\section{ARTICLE SUMMARY}

Article focus

Non-steroidal anti-inflammatory drugs (NSAIDs) and proton pump inhibitors (PPIs) are two types of drugs that are closely associated with opposite risk of peptic ulcer bleeding. Their relation to occurrence, recurrence and death of bleeding in the population level is not clear.

Key messages

- The sales of NSAIDs and PPIs (inversely) correlate with recurrence and death after peptic ulcer bleeding on the population level.

- Women appeared to have a higher fatality of bleeding which might be associated with older age and higher sales of NSAIDs.

- The results highlight the need for careful consideration of drug prescriptions to patients with peptic ulcer bleeding.

Strengths and limitations

- Drug sales/use and peptic bleeding outcomes could not be linked with regard to individual patients.

- Re-bleeding could only be identified on the basis of re-admission, which means we might have lost information regarding re-bleeding that occurred within the same case of hospitalisation.

- It is difficult to find a suitable cut-off day for definition of re-bleeding.

Re-bleeding and death after peptic ulcer bleeding occur in $7-16 \%$ and $3-14 \%,{ }^{2} 56$ respectively. These figures might increase as a result of the increasing average age of many populations. ${ }^{1278}$ The high risk of recurrence and death highlights the need to identify the best preventive measures available. The established risk factors for peptic ulcer bleeding include $H$ pylori infection and medications such as non-steroidal anti-inflammatory drugs (NSAIDs), whereas proton pump inhibitors 
(PPIs) can prevent ulcer bleeding. ${ }^{9}$ We aimed to examine how the sales of PPIs and NSAIDs correlate with the incidence, recurrence and death of peptic ulcer bleeding from a population perspective.

\section{METHODS}

\section{Study design}

This was a nationwide ecological study that addressed the correlation between relevant drug sales and peptic ulcer bleeding in Sweden during the period 2000-2008. We used complete Swedish nationwide registers to collect data on sales of NSAIDs and PPIs, hospitalisation and death after peptic ulcer bleeding. The average daily defined doses (DDDs) of NSAIDs and PPIs were compared with the incidence, recurrence within 60 days after hospitalisation for bleeding, and 30-day death after admission for peptic ulcer bleeding, in Sweden. The Regional Ethics Committee in Stockholm approved the study.

\section{Data collection}

Aggregated data on drug sales in Sweden during the study period were available from the Swedish Prescribed Drug Register. This register records all prescribed and collected medications in the entire Swedish population of approximately 9 million inhabitants. ${ }^{10}$ The Prescribed Drug Register contains data on the age and sex of patients together with the names of prescribed drug substances according to the anatomical therapeutic chemical (ATC) classification. All NSAIDs (ATC codes: M01A) and PPIs (ATC codes: A02BC and A02BD) were used for this study. All NSAIDs with ATC codes of M01A were sold as prescription drugs except a few types of ibuprofen in Sweden.

Patients with peptic ulcer bleeding were identified from the Swedish Patient Register, which contains complete, nationwide data on all codes representing diagnoses and surgical procedures relating to inhospital care in Sweden since 1987. Codes representing peptic ulcer bleeding according to the international classification of diseases V.10 were used (K25.0, K25.4, K26.0, K26.4, K27.0, K27.4, K28.0, K28.4, K92.0, K92.1 and K92.2). Since the treatment of ulcer perforation is different from the treatment of ulcer bleeding, patients with perforation were excluded. Re-admission for peptic ulcer bleeding within 1 day of discharge was not regarded as a new case of bleeding. Re-bleeding was defined as an episode of bleeding that occurred within 60 days after a previous bleeding. Death was defined as any death occurring within 30 days of the date of admission for peptic ulcer bleeding. Death dates were obtained from the Death of Cause Register and the Swedish Population Register. The personal number, which is the unique identity for all the Swedish residents, was used to link data among different registers.

\section{Statistical analyses}

Average DDD and time trends regarding the sales of PPIs and NSAIDs were calculated on the basis of the average population for each year. DDD/TID was described as DDDs/thousand inhabitants/day. A linear regression model was applied to test the statistical significance of trends at the 5\% level. Correlation analyses were performed between drug sales and the incidence, recurrence and death of peptic ulcer bleeding. All analyses were gender-specific. Figures were plotted to show the correlations between drug sales and bleeding events. All statistical analyses were performed using SAS V.9.2 (SAS Institute, Cary, North Carolina, USA).

\section{RESULTS}

\section{Trends of PPI and NSAID sales}

The sales of PPIs increased during the study period, except for a temporary drop in 2003 (figure 1). The sales of NSAIDs increased until 2004, after which there was a decrease to a level lower than in the year 2000 (figure 2). The decreased NSAID sales were particularly evident in patients over 75 years of age (data not shown). Women bought more PPIs and NSAIDs than men (figures 1 and 2), and this difference was more
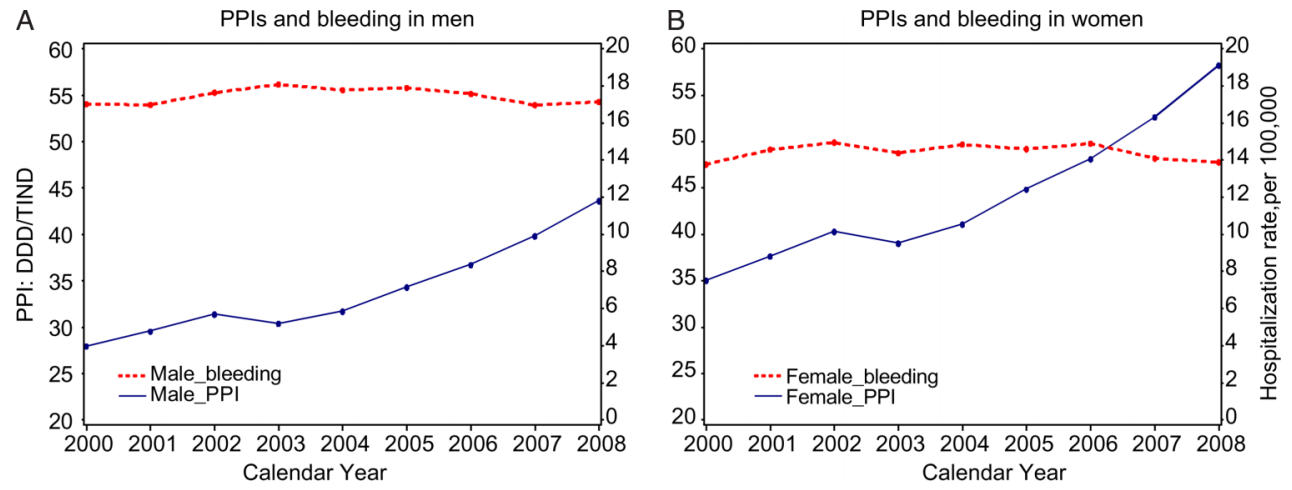

Figure 1 Sales of proton pump inhibitors (PPIs) in daily defined doses (DDDs)/1000 inhabitants/day and hospitalisation rate for peptic ulcer bleeding (hospitalisations for bleeding/100 000 inhabitants) in Sweden in 2000-2008. (A and B) Represent men and women, respectively. 

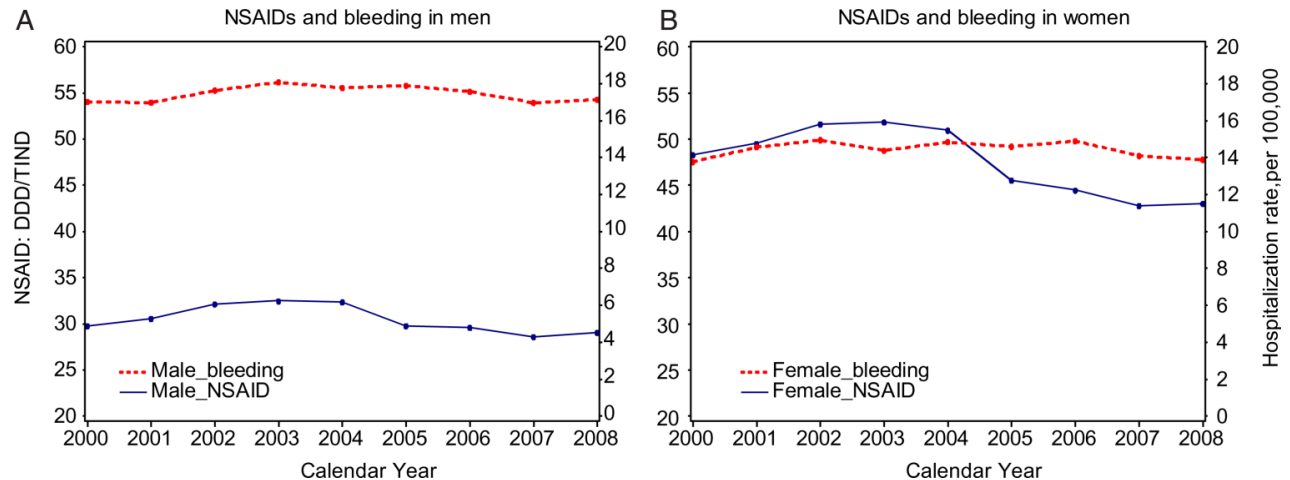

Figure 2 Sales of non-steroidal anti-inflammatory drugs (NSAIDs) in daily defined doses (DDD)/1000 inhabitants/day and hospitalisation rate for peptic ulcer bleeding (hospitalisations for bleeding per 100000 inhabitants) in Sweden in 2000-2008. (A and B) Represent men and women, respectively.

obvious with regard to NSAIDs. We also analysed the trend of sales of aspirin and $\mathrm{H}_{2}$ receptor antagonists (data not shown) which seems not very relevant with trends of peptic ulcer bleeding, then we focused on correlations of PPIs/NSAIDs and peptic ulcer bleeding in this study.

Incidence, recurrence and death of peptic ulcer bleeding The hospitalisation rate for peptic ulcer bleeding was stable during the study period, although a higher rate was observed in men than in women (figure 1). The rate of recurrence of bleeding was similar between the genders, although the recurrence rate in women showed a slightly decreasing trend (figure 3). Thirty-day death after peptic ulcer bleeding decreased during the study period, especially in men (figures 5 and 6). Furthermore, women showed a higher death rate in different time periods (figure 6).

\section{PPIs and NSAIDs sales and peptic ulcer bleeding}

The trend of peptic ulcer bleeding did not correlate with PPIs sales in either gender (figure $1 ; R_{\text {male }}=-0.2274$, $\left.\mathrm{P}_{\text {male }}=0.5562, \mathrm{R}_{\text {female }}=-0.2398, \mathrm{P}_{\text {female }}=0.5342\right)$, but it correlated marginally with the trend of the sales of NSAIDs in men only (figure 2; $\mathrm{R}_{\text {male }}=0.6571, \mathrm{P}_{\text {male }}=0.05$, $\left.\mathrm{P}_{\text {female }}=0.2633, \mathrm{R}_{\text {female }}=0.4177\right)$.

\section{PPI and NSAID sales and peptic ulcer re-bleeding}

The time trends of re-bleeding did not correlate with the sales of PPIs or NSAIDs in men (figures 3 and 4, $\left.\mathrm{R}_{\text {male }}=0.2227, \mathrm{P}_{\text {male }}=0.5647 ; \mathrm{R}_{\text {female }}=0.023, \mathrm{P}_{\text {female }}=0.9522\right)$, but the decreased occurrence of re-bleeding in women correlated with the time trends of both PPI sales $\left(R_{\text {male }}=\right.$ $\left.-0.8754, \mathrm{P}_{\text {male }}=0.002\right)$ and NSAID sales $\left(\mathrm{R}_{\text {female }}=0.7161\right.$, $\left.\mathrm{P}_{\text {female }}=0.03\right)$.

\section{PPI and NSAID sales and 30-day death}

There was an inverse correlation between PPI sales and death in both genders $\left(R_{\text {male }}=-0.9392, P_{\text {male }}=0.0002\right.$, $\mathrm{R}_{\text {female }}=-0.8561, \mathrm{P}_{\text {female }}=0.003$; figure 4 ), and the NSAID sales showed a close correlation with death of bleeding in both genders $\left(\mathrm{R}_{\text {male }}=0.7278, \mathrm{P}_{\text {male }}=0.03, \mathrm{R}_{\mathrm{female}}=0.7858\right.$, $\mathrm{P}_{\text {female }}=0.01$; figure 5).

\section{DISCUSSION}

This study indicates that sales of NSAIDs and PPIs (inversely) correlates with 30-day death (both sexes) and recurrence (womenfemales) of peptic ulcer bleeding in
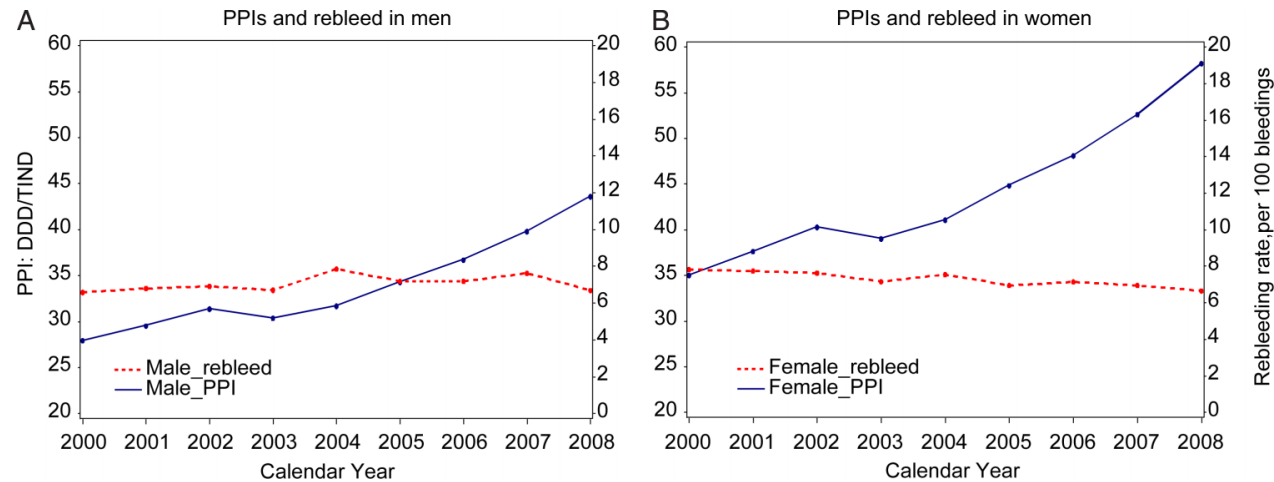

Figure 3 Sales of proton pump inhibitors (PPIs) in daily defined doses (DDDs)/1000 inhabitants/day and peptic ulcer re-bleeding rate within 60 days (number of re-bleeders/100 inhospitalisations for bleeding) in Sweden in 2000-2008. (A and B) Represent men and women, respectively. 



Figure 4 Sales of proton pump inhibitors (PPIs) in daily defined doses (DDDs)/1000 inhabitants/day and 30-day death of peptic ulcer bleeding (number of deaths within 30 days/100 hospitalisations for bleeding) in Sweden 2000-2008. (A and B) Represent men and women, respectively.

the unselected population. Women appeared to have a higher death of bleeding which might be associated with age and greater sales/use of NSAIDs.

The main strength of this study is the nationwide, complete data collection regarding drug sales, hospitalisation for bleeding and death. Since there is virtually no private care for peptic ulcer bleeding in Sweden and since such bleeding usually requires hospitalisation, the incidence of bleeding and re-bleeding covered by this study should represent population-based figures. There are, however, several weaknesses that should be acknowledged. Drug sales/use and peptic bleeding outcomes could not be linked with regard to individual patients. This ecological design makes the interpretations more uncertain. Re-bleeding could only be identified on the basis of re-admission, which means we might have lost information regarding re-bleeding that occurred within the same case of hospitalisation. On the other hand, it is difficult to find a suitable cut-off day for definition of re-bleeding. The definition of death of bleeding was based on death within 30 days after discharge, since it is difficult to assess if death actually results from bleeding. Furthermore, selection bias for PPIs users could exist since PPIs were also prescribed for gastroesophageal reflux diseases. This might dilute the correlation between PPIs and incidence of peptic ulcer bleeding. Nevertheless, we also have studied correlation between PPIs/NSAIDs and re-bleeding, PPIs/NSAIDs and mortality after bleeding diagnosis which may possibly further pinpoint the specific correlation between PPIs/NSAIDs and peptic ulcer bleeding. In addition, a few types of ibuprofen as one of NSAIDs were sold as over-the-counter drugs which might lead to selection bias for NSAIDs in this study. Since most NSAIDs were prescribed drugs and actually few persons buy drugs without prescriptions in Sweden due to the nationwide healthcare system, this selection bias may be negligible.

NSAIDs constitute an established risk factor for peptic ulcer bleeding, but the sales/use of these medications at the population level is less well-documented. A previous study reported that the sales of NSAIDs in Sweden increased during the period 1978-2002. ${ }^{11}$ The present study shows that sales have decreased since 2004 with regard to both genders, and this decrease is more obvious in women and in the population aged over 75 (data not shown). This decline might contribute to the decreasing trend of peptic ulcer death and re-bleeding in women. Similarly, the increased sales/use of PPIs
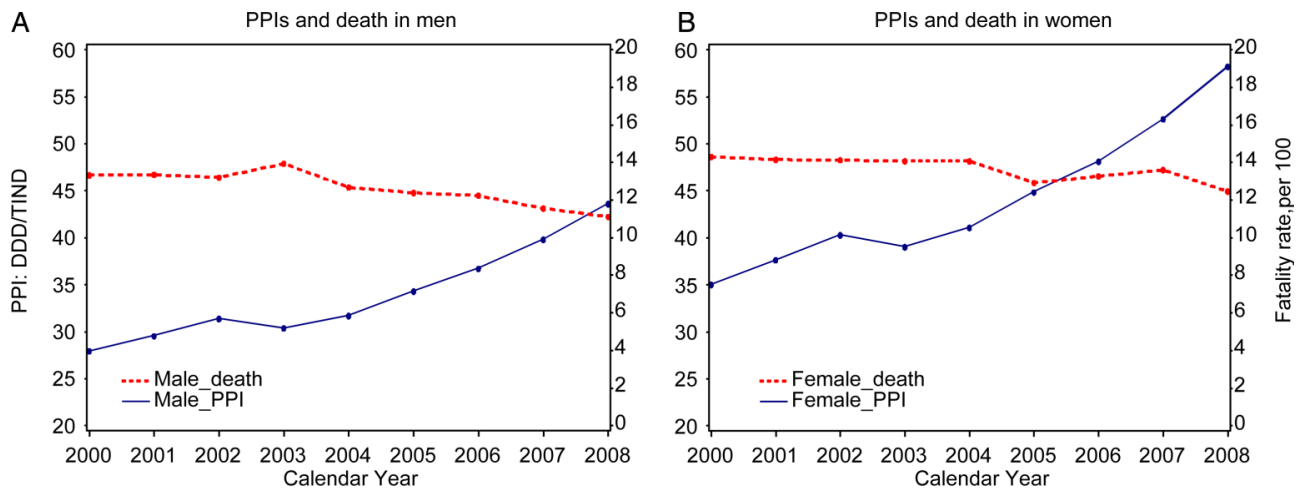

Figure 5 Sales of non-steroidal anti-inflammatory drugs (NSAIDs) in daily defined doses (DDDs)/1000 inhabitants/day and 30-day death of peptic ulcer bleeding (number of deaths within 30 days per 100 hospitalisations for bleeding) in Sweden in 2000-2008. (A and B) Represent men and women, respectively. 

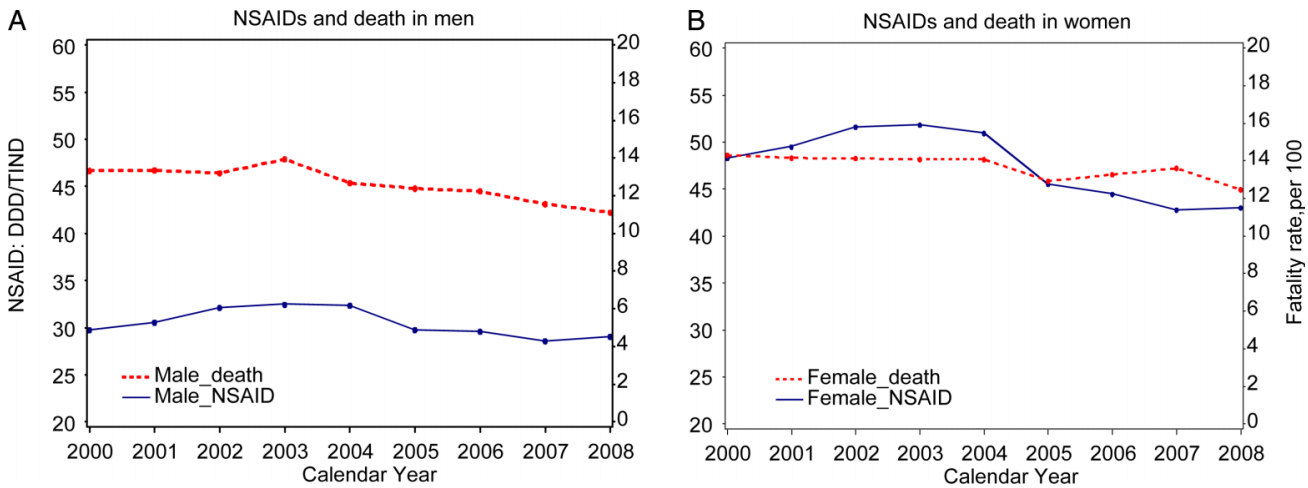

Figure 6 Sales of non-steroidal anti-inflammatory drugs (NSAIDs) in daily defined doses (DDD)/1000 inhabitants/day and peptic ulcer re-bleeding rate within 60 days (number of re-bleeders/100 hospitalisations for bleeding) in Sweden in 2000-2008. (A and B) Represent men and women, respectively.

might contribute to these trends. The correlation between sales/use of PPIs and death indicates that PPIs might prevent deaths from bleeding. This relation is more apparent in the male population, which shows a very interesting, coinciding pattern. The different correlations of drug and bleeding death between men and women could be attributed to the different sales/use of PPIs and NSAIDs between the genders. Previous studies have found that PPIs may help reduce peptic ulcer bleeding, re-bleeding and the need for surgery, but not death. $^{12-14}$ It is difficult to differentiate between the respective roles of PPIs and NSAIDs. Nevertheless, the gastric mucosal protective effects of PPIs could be beneficial if NSAIDs are used. Surprisingly, PPIs are used in only $10-22 \%$ of patients admitted with a peptic ulcer disease and using NSAIDs. ${ }^{15-17}$ Our recent study, using individual prescription data from Sweden, revealed that less than $40 \%$ long-term NSAIDs users (those prescribed NSAIDs for more than 180 days) were prescribed concomitantly gastroprotective medication. ${ }^{18}$ Furthermore, this study found that men took less gastroprotective drugs compared with women, despite the fact that concomitant antithrombotic treatment was more common in men. On the other side, a German study found that overprescription of drugs might increase risk of adverse effect because of drug-drug interactions. ${ }^{19}$ It may indicate that the use of NSAIDs in high-risk population, for example, patients with a history of complicated peptic ulcer and elderly population with several concomitant prescriptions, should be cautious, and a personalised regimen should be considered thoroughly.

The incidence of peptic ulcer bleeding is higher in men than in women, but few studies have reported gender distribution with regard to re-bleeding and death. One British case series study suggested that women are at a higher risk of perforation or death than men. ${ }^{20}$ The older mean age in women was, however, an important factor in that study, and this is consistent with our data (data not shown). The greater sales/use of NSAIDs in women cannot be ignored. The rapid decrease of NSAID sales/use in women, since 2004, might contribute to the significantly decreased occurrence of re-bleeding and death in this group in which PPI sales/use is increasing continuously.

In conclusion, although the sales/use of NSAIDs and PPIs in the general population does not seem to mirror the incidence of peptic ulcer bleeding, such sales/use correlate with re-bleeding and death of peptic ulcer bleeding. This correlation seems more obvious in old women which proposes an intriguing issue for future study. The potential reduced risk of death due to decreased use of NSAIDs (especially in old women) and increased use of PPIs (especially in men) warrants further investigation.

\section{Author affiliations}

${ }^{1}$ Upper Gastrointestinal Research, Department of Molecular Medicine and Surgery, Karolinska Institutet, Stockholm, Sweden

${ }^{2}$ Section of Upper Gastrointestinal Surgery, Department of Surgery, South General Hospital, Stockholm, Sweden

${ }^{3}$ Centre for Epidemiology, The National Board of Health and Welfare, Stockholm, Sweden

${ }^{4}$ King's College London, London, UK

Contributors $Y L, R L$ and $J L$ were responsible for the design of the study. $\mathrm{RL}$ was responsible for acquisition of the data. $\mathrm{YL}$ was responsible for data analysis including arrangement of tables and graphs. ES, CS, RL and JL provided input into data analysis and all the authors contributed to interpretation of the results. YL initiated the draft of the manuscript and then circulated it repeatedly among all the authors for critical revision.

Funding Funded by the Swedish Research Council and the Astrid and David Hagelén Foundation.

Competing interests None.

Ethics approval The Regional Ethics Committee in Stockholm.

Provenance and peer review Not commissioned; externally peer reviewed.

Data sharing statement The database was ordered from the Swedish National Board of Health and Welfare. The data are sensitive and we cannot share the data on the website. The data will be stored in Karolinska Institutet for more than 5 years for further check-up. If anyone is interested in the data, it might be possible to apply from http://www.socialstyrelsen.se/.

\section{REFERENCES}

1. Blatchford O, Davidson LA, Murray WR, et al. Acute upper gastrointestinal haemorrhage in west of Scotland: case ascertainment study. BMJ 1997;315:510-14 
2. van Leerdam ME, Vreeburg EM, Rauws EA, et al. Acute upper GI bleeding: did anything change? Time trend analysis of incidence and outcome of acute upper Gl bleeding between 1993/1994 and 2000. Am J Gastroenterol 2003:98:1494-9.

3. Longstreth GF. Epidemiology of hospitalization for acute upper gastrointestinal hemorrhage: a population-based study. $A m \mathrm{~J}$ Gastroenterol 1995;90:206-10.

4. Yavorski RT, Wong RK, Maydonovitch C, et al. Analysis of 3294 cases of upper gastrointestinal bleeding in military medical facilities. Am J Gastroenterol 1995;90:568-73.

5. Vreeburg EM, Snel P, de Bruijne JW, et al. Acute upper gastrointestinal bleeding in the Amsterdam area: incidence, diagnosis, and clinical outcome. Am J Gastroenterol 1997;92:236-43.

6. Hearnshaw SA, Logan RF, Lowe D, et al. Acute upper gastrointestinal bleeding in the UK: patient characteristics, diagnoses and outcomes in the 2007 UK audit. Gut 2011;60:1327-35

7. Paspatis GA, Matrella E, Kapsoritakis A, et al. An epidemiological study of acute upper gastrointestinal bleeding in Crete, Greece. Eur J Gastroenterol Hepatol 2000;12:1215-20.

8. Barkun A, Sabbah S, Enns R, et al. The Canadian Registry on Nonvariceal Upper Gastrointestinal Bleeding and Endoscopy (RUGBE): Endoscopic hemostasis and proton pump inhibition are associated with improved outcomes in a real-life setting. $A m \mathrm{~J}$ Gastroenterol 2004;99:1238-46.

9. Leontiadis GI, Sharma VK, Howden CW. Systematic review and meta-analysis of proton pump inhibitor therapy in peptic ulcer bleeding. BMJ 2005;330:568.

10. Wettermark B, Hammar N, Fored CM, et al. The new Swedish Prescribed Drug Register-opportunities for pharmacoepidemiological research and experience from the first six months. Pharmacoepidemiol Drug Saf 2007;16:726-35.
11. Hermansson M, Ekedahl A, Ranstam J, et al. Decreasing incidence of peptic ulcer complications after the introduction of the proton pump inhibitors, a study of the Swedish population from 1974-2002. BMC Gastroenterol 2006;25:CD002094.

12. Leontiadis GI, Sharma VK, Howden CW. WITHDRAWN: proton pump inhibitor treatment for acute peptic ulcer bleeding. Cochrane Database Syst Rev 2006;25:CD002094.

13. Ray WA, Murray KT, Griffin MR, et al. Outcomes with concurrent use of clopidogrel and proton-pump inhibitors: a cohort study. Ann Intern Med 2010;152:337-45.

14. Zed PJ, Loewen PS, Slavik RS, et al. Meta-analysis of proton pump inhibitors in treatment of bleeding peptic ulcers. Ann Pharmacother 2001;35:1528-34.

15. Di Fiore F, Lecleire S, Merle V, et al. Changes in characteristics and outcome of acute upper gastrointestinal haemorrhage: a comparison of epidemiology and practices between 1996 and 2000 in a multicentre French study. Eur J Gastroenterol Hepatol 2005;17:641-7.

16. Ohmann C, Imhof M, Ruppert C, et al. Time-trends in the epidemiology f peptic ulcer bleeding. Scand J Gastroenterol 2005;40:914-20.

17. Johnell K, Fastbom J. Concomitant use of gastroprotective drugs among elderly NSAID/COX-2 selective inhibitor users: a nationwide register-based study. Clin Drug Investig 2008;28:687-95.

18. Ljung R, Lu Y, Lagergren J. High concomitant use of interacting drugs and low use of gastroprotective drugs among NSAID users in an unselected elderly population: a nationwide register-based study. Drugs Aging 2011;28:469-76.

19. Ahrens D, Chenot JF, Behrens G, et al. Appropriateness of treatment recommendations for PPI in hospital discharge letters. Eur J Clin Pharmacol 2010;66:1265-71.

20. Smart HL, Langman MJ. Late outcome of bleeding gastric ulcers. Gut 1986;27:926-8. 